\title{
Qualitative Expert Views' on the Feasibility of Tele-Counseling Services in Malaysia
}

Bakar, A. Y. A. ${ }^{\bowtie}$ (iD

\section{Mejah, H. ${ }^{2}$}

Amat, S. $^{3}$

${ }_{1,2,3}$ Faculty of Education, Universiti Kebangsaan Malaysia, Bangi, Selangor, Malaysia.

${ }^{2}$ Email:yazid3338@ukm.edu.myTel:+60192246917

¿Email:izah_gurls8@yahoo.com Tel:60192538676

sEmail: sallehamat@gmail.com Tel: +60193327s94

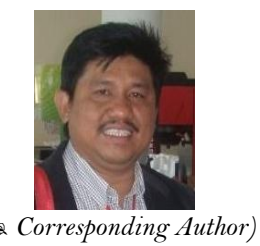

\begin{abstract}
Online counseling is a delivery of therapeutic interventions in cyberspace where there are communication between trained professional counselors and clients using electronic communication technologies such as telephone, computer, email, internet as mediators in separate places. The provision of counseling services via telephone (also known as tele-counseling) is relatively new and the guiding policies are still being drafted by the local governing body of counseling profession. Hence, the main objective of this qualitative study is to explore expert perceptions of the feasibility of counseling provision via telephone, also known as tele-counseling. A total of five local experts in the field of counseling were involved as the respondents of this study. Based on the thematic analysis of the data, there were three main themes generated from the objectives of this study: (i) acceptance of tele-counseling services, (ii) advantages of telecounseling services and (iii) limitations of tele-counseling services. The findings suggest that telecounseling should be considered as an alternative to the conventional face-to-face counseling services in Malaysia. However, despite all the advantages it may offer, counselors who intend to engage in providing this service must also consider the potential limitations of its implementation.
\end{abstract}

Keywords: Counseling, Expert views, Feasibility study, Tele-counseling, Qualitative research, Malaysia.

Citation | Bakar, A. Y. A; Mejah, H; Amat, S (2020). Qualitative Expert Views' on the Feasibility of Tele-Counseling Services in Malaysia. Journal of Education and e-Learning Research, 7(3): 270276.

History:

Received: 20 May 2020

Revised: 14 July 2020

Accepted. 7 Angust 2020

Accepted: 7 August 2020

Licensed: This work is licensed under a Creative Commons

Attribution 3.0 License (c)

Publisher: Asian Online Journal Publishing Group
Acknowledgement: All authors contributed to the conception and design of the study.

Funding: This study received no specific financial support

Competing Interests: The authors declare that they have no conflict of interests.

Transparency: The authors confirm that the manuscript is an honest, accurate, and transparent account of the study was reported; that no vital features of the study have been omitted; and that any discrepancies from the study as planned have been explained.

study as planned have been explained.
Ethical: This study follows all ethical practices during writing.

\section{Contents}

1. Introduction

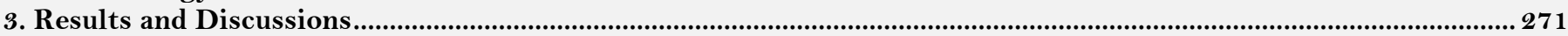

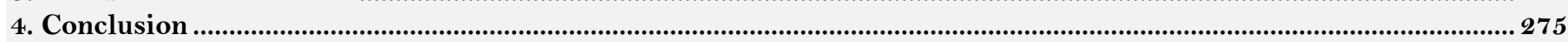

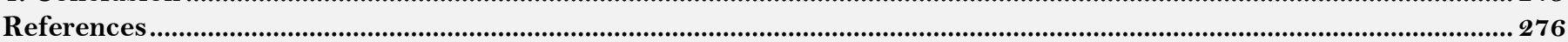




\section{Contribution of this paper to the literature}

This research contributes to the study of tele-counseling provision in Malaysia. Since the guiding policies to this new provision of online counseling service is still lacking, this research will enrich the current existing literatures; thus, contributes to the establishment of such policies in the future.

\section{Introduction}

The advent of the information age or the digital world has begun to permeate the general public as a package that is impossible to be separated from our lives (Mohd Nor, 2000). Advances in science and technology especially in the field of information and communication technology will undoubtedly influence our day-to-day environment, innovation and reform, as well as in the field of counseling (Ithnin \& Ibrahim, 2000).

The first online help desk service is known as online counseling, virtual counseling and e-counseling. Online counseling is a new way of enabling a helping relationship to occur quickly and effectively through the medium of telephone, computer and internet. According to American Counseling Association (1999) online counseling is defined as

"Use of electronic communications and computer technology in the counseling process in general over the internet."

The official definition provided by the Bloom (1997) is a process of professional counselling that occurs when clients and counselors are in different places and use technology to communicate. Richards and Vigan'o (2012) explained that online counseling is a delivery of therapeutic interventions in cyberspace where communication between trained professional counselors and clients uses electronic communication technologies such as telephone, computer, email, internet as mediators in separate places.

Based on these definitions, it can be concluded that counseling can not only be done face-to-face. It can also be done online and does not require clients and counselors to appear face to face. Online counseling is a process of counseling that is no longer limited to a two-point meeting in a room where clients no longer have to meet with a counselor, in the same room and at the same time to get counseling services. However, this definition may be subject to change or additional depending on current developments. This service is not intended to replace psychotherapy face-to-face, but is useful as an alternative source of assistance in addressing life issues.

In Malaysia, online counseling services are on the rise and are gaining attention (Mejah, Bakar, \& Amat, 2019). It is even considered as one of the new alternatives for counseling practitioners in providing help for the community (Mejah et al., 2019). However, there are still conventional-thinking counseling practitioners who criticize online counseling to not be a real counseling service.

This is because; the use of technology in counseling services has led to no face-to-face counseling sessions as conventional methods of counseling (Civin, 2000; Nor Ba'yah \& Yahaya, 2001; Rosenfield, 2002). In fact, online counseling is considered as a practice that is out of the ordinary practice of counseling, which eventually undermine the purity of conventional counseling that has been a practice among counselors since (Mejah et al., 2019). Counselors are also concerned that the spread of online counseling will change the authenticity of the counseling practice itself (Mejah et al., 2019). These concerns have caused some counselors to shut down and refuse to accept changes and reforms (Mejah et al., 2019). It may also mean trying to push the pace of change and technological development in counseling services (Nor Bayah \& Yahaya, 2001). The purpose of this study is to explore the perceptions of experts in the field of counseling in Malaysia on the performance of tele-counseling services in Malaysia

\section{Methodology}

This study is a qualitative research design using a case study approach that involves in in-depth interviews using flexible structured interview instruments. The choice of case study approach for this study is to obtain clear, detailed and in-depth information and to produce more accurate descriptions of the study (Creswell, 2014; Yin, 2014). The sample used in this study are experts in the field of counseling in Malaysia. Among the sample criteria are: (i) Registered Counselors, (ii) Senior Members of the Malaysian Board of Counselors (MOH) and the Malaysian Association of Counselors (PERKAMA) and (iii) involved in the formulation of policy and counseling act in Malaysia. In-depth interviews with five respondents for this study were selected based on the sample criteria.

The analysis of this study wass done through the process of data preparation until interpretation. The data was categorized into related themes used to interpret the data to obtain information based on the objectives of this study. The process of analyzing this data was conducted using Nvivo 12 software which, as in line with Glaser. and Peshkin (1992)'s view, can help researchers use qualitative methods to effectively organize, analyze and store data. This study emphasized systematic steps in analyzing data-free and dependent-mode data (Dini, 2016; Glaser \& Strauss, 1967). Therefore, specific themes and codes were provided to all relevant information and analyzed based on the data collected in response to the research objectives. Finally, the analysis of the research was based on the theme of the interview from the objective of the study, which was to explore the perceptions of experts in the field of counseling in tele-counseling services in Malaysia. All of the themes selected in this study were the best and can be interpreted using a narrative approach of all the issues presented (Suriati \& Colonius, 2008).

\section{Results and Discussions}

Based on the results of thematic data analysis of the research, there were three themes generated from the objectives of this study: (i) acceptance of tele-counseling services, (ii) advantages of tele-counseling services and (iii) limitations of tele-counseling services.

\subsection{Acceptance of Tele-Counseling Services}

Findings on expert perceptions of the viability of tele-counseling services in Malaysia from the point of view of most of the respondents stated that tele-counseling is not real counseling based on the purpose of counseling, which is the process of helping through face-to-face communication between counselor and client until the client 
has come to his or her senses. $\mathrm{R} 4$ stated that real counseling is a counseling session that needs to be conducted face-face between counselor and client in one place,
.....to me, the real counseling is face-to-face, so, when going on phone, is not a real counseling. Why? Because when you run a session on the phone, it won't happen face-to-face, right? We should go back to the original meaning of counseling. To me, it is not a counseling session. Okay, as I said to you before, a counseling session should be done face-to-face. If not, then it cannot be called as counseling. Of course, to me, I disagree. I disagree with tele-counseling.....
...It is undoubtedly true that technology is a need, but it is different case in counseling process as if we were to hold on to the real meaning of counseling which is to go through the process face-to-face then it is relevant. It can be applied if it's like a video call, because through that method, counselor and client are able to see each other. So, it can be practiced, however for me, to just listening is not counseling. And I would prefer if the video can show the whole body of the counselor and client themselves. So, it can be practiced. For me, through phone, I can see that it is just no more than something a client will use to make appointment or just as the beginning stage of a counseling session to occur....

This acceptance was also in line with R2's acceptance of tele-counseling, stating that tele-counseling is an early-stage help process and that it is more about the intervention and consultation clients need such as making appointments and seeking guidance.

.... $i$ am based on what has been stated in the act. If we look at the traditional definition of counseling, it means face-to-face but the technology has been used as an intervention. This is probably because of the distance, so it is better than nothing and acceptable for the first stage, as it is still considered as helping process but not really a real counseling because even in the act, there is not concept of charging your client for the service when you do tele-counseling because there is no charge and fees approved. Even in our country also it is not stated anywhere that we can charge if the client wants to use tele-counseling. So, the definition of counseling is a process that we can say to be face-to-face. That is what on the surface but I don't really object that we cannot help individuals through any other medium, we can help but it is not something we call as the real counseling session per se......

..... Tes, it is true that we know that the client has problem but even if a counselor proceed with the counseling session, but for me the counselor can do that but we won't claim it as doing a counselling. This is because in counseling service, if we do any counseling session, we have to do it properly through correct medium and such. One, you can do the consultation, psychology consultation, another one is maybe we can say it as psychological intervention. But the technique that we use is like intervention, something like we give advice or opinion....

Similarly, the R3 acceptance also stated that tele-counseling services are an intermediary between counselors and clients for making face-to-face meetings and for counselors to obtain client's initial information before a faceto-face session.
... Like the psychology department itself, it offers e-counseling via email, but rather a summary of the issues it wants to address. People say more about whether this is an intro or whatnot. And usually clients will tell us a little bit about the issues they are facing, and we will contact them to ask them to come face-to-face to the session according to the time available for both of us. That is it and if any other session through phone, it is more to something considered to be the first counseling or the first session to attract clients to see a counselor to conduct a counseling session there (at the counselor's place) ...
...to me, for this counseling, we stick to the original meaning of counseling. The process of helping face to face between counselor and clients to achieve common sense. I stick to the term "Face to face". That's my advice. But I don't know anyone else... in my own office as I said earlier, we only used the phone for appointments and intro or just to get information on client issues, but for the real session we would do it face-to-face...

R4 acceptance on the other hand mentioned that the effectiveness of using technology as the counselor and client's medium in conducting counseling sessions was less than the conventional, face-to-face sessions. The presence of non-verbal communication is vital in counseling and it is said that face-to-face counseling is better because counselor can see the client face-to-face and able to monitor the non-verbal communication with clients and it is one of the most important parts in the counseling process. Likewise, the client can feel the presence of the counselor in assisting the counseling session.

... when I was in the oversea, I was not exposed to tele-counseling, because as I was there what I see is I think they still believe in face to face counseling. If you were doing tele-counseling through the internet, skype or whatnot I guess I'm not going to do it. Like I said earlier, I am more to face to face counseling because I feel that when I do counseling, first thing I want to do is to see my client and I want my client to see me as well and observation is very important. One of the things that is very important is to see if he has non-verbal reaction, to hear his voice, to know what the impact is of my presence to him. Yes the pressure is there. If it is done through skype or through other medium, I feel like it is not 100\% done. It surely can be done but to be frank, I 
will not do it....

The overall response to the tele-counseling service was that the most of the respondents stated that telecounseling service was not real counseling and that it was only a mediation between counselors and clients for the first-aid process and that most of the respondents still adhered to the original concept of counseling which requires counselors and clients to meet for the helping relationship. The statement was consistent with Boy and Pine (1968) statement explaining that counseling is a face-to-face relationship in which a client will seek the help of a counselor. These relationships include components such as effective communication, mutual respect, genuine client acceptance by the counselor and focuses on client's need, concerns and feelings.

\subsection{Advantages of Tele-Counseling Services}

On the other hand, most of the respondents agreed that technology is a necessity today, and that the use of phones today has also become a necessity. R 1 stated that the use of technology can be applied as long as it adheres to the original purpose of face-to-face counseling such as the use of video calls that are still advisory where clients can see each other. However, the phone usage is still relevant if the client wants to make an appointment and considered as a help process at an early stage which channel aid for clients who require immediate help in resolving the problem they are facing. However, for the next session, the counselor should play a role in attracting clients to run the session face to face.
..It is undoubtedly true that technology is a need, but it is different case in counseling
process as if we were to hold on to the real meaning of counseling which is to go
through the process face-to-face then it is relevant. It can be applied if it's like a
video call, because through that method, counselor and client are able to see each
other. So, it can be practiced, however for me, to just listening is not counseling.
And I would prefer if the video can show the whole body of the counselor and client
themselves. So, it can be practiced. For me, through phone, I can see that it is just no more than something a client will use to make appointment or just as the beginning stage of a counseling session to occur....
...Ok, it is no doubt that telephone is a necessity. So, just like what I have said to you,
if it is only for the first stage of counseling session then it is fine because for these individuals who have problems, he or she will think on how to solve the problem on the spot and they would not think of other things. So, it is applicable to use phone to ease their mind that really need some help by that time as a start and also to make appointment. So, as a professional counselor, you need to know how to attract your client to come and meet you face-to-face for the next session. For the first session, it is okay for the counselor to listen to the client to soothe out his problem but the client should also meet face-to-face with the counselor so that the counselor can see the non-verbal communication gestures just like what have been learnt in the counseling skills itself...

Similarly, $\mathrm{R} 2$ cited the telephone as a prerequisite to the crisis of intervention, as a first-aid intervention in building rapport with clients who needed immediate assistance in relieving issues that were affecting clients at the time, such as depressed clients. However, for further assistance, clients still need to come face to face with the counselor.

...so, there are individuals who need help and feel depressed or whatnot, then they call through phones, skype or also through other medium then it is fine, but for the next level is that individual should come and meet us to build first rapport and whatnot before he or she really comes for the next session and meet us. This is more to of what we call crisis of intervention...(R2)

... if the depressed person wants to run away from home and so on, the issue will be he will call first, releases what is inside him first and the he goes for real counseling. For example, for distance initial stage, instead of not helping then it is better to offer help, it's better. On the spot, you can make a call, skype and of course through these medium, I can see some impact rather than nothing at all in terms of help... (R2)

The opinion was supported by R3 saying that it was an advantage for individuals who could not meet face to face with the counselor due to obstacles such as long distances, or even facilitated individuals who feel embarrassed to talk about sensitive issues.

... maybe it is an advantage for clients who are not able to meet face to face, unable to meet the counselor because of distance constraints, living in remote areas. There are also, those who feel embarrass to talk about private issues probably sensitive cases involving their own dignity such as rape, incest, or any other embarrassing cases that can raise up any issue, it probably gives some kind of advantage to them. But for me, it can be practiced if the counselor is capable and experienced in handling telecounseling. Because of what, if let say the client has complex issues which requires high skills for it to be solved and takes up a lot of time, my advice would be that it is not suitable to be solved through phone...(R3)

The findings were in line with Poh, Jaladin, and Abdullah (2013) findings, that online counseling has the potential to assist clients with problems in obtaining counseling services for clients located in remote areas. In addition, counseling through phone can help in some cases and it provides opportunity for shy individuals to share sensitive issues who are at the same time want to protect their identity from being recognized as stated by $\mathrm{R} 4$.

..it can somehow be done over the phone. Like before, I used to get a call from this one girl who often listens to me on the radio. She knows that I was a director of social welfare department back then in Johor and I was always invited to give talk 
on the radio that time. In her case, it involved incest case. I know her name but I never meet her. I don't know how she gets my home phone number. She told me that she was raped by her own brother. She was so stressed out thinking that she is no longer a virgin and need someone to help her out but she doesn't want people to know who she is. Then only I recall, maybe for some cases, it can be done through phone calls...

(R4).

Ramsden (2011) stated that the use of online counseling services is very helpful for clients who require a safe environment to communicate emotional issues to counselors. In other words, clients feel more secure and comfortable (Leibert, Archer, Munson, \& York, 2006; Ramsden, 2011; Young, 2005) to share with counselors about personal issues involving emotions without having to face them. This is in line with Hanley (2009) online counseling is where counselors and clients seek a more comfortable environment during counseling sessions without having to do face-to-face counseling between counselors and clients.

Based on an online session management method that does not require visual self-disclosure (Pelling, 2009; Poh et al., 2013) found that adequate online-only sessions can provide a very comfortable sharing space for clients to share especially for very sensitive issues like adultery, incest, pedophilia, abuse victims, sexual orientation, drug addicts, drug traffickers or even those involving criminal or crime related issues etc.

\subsection{Limitation of Tele-Counseling Services}

The use of telephone technology is now widespread and is a necessity in all areas including counseling services. It is undeniable that the presence of various telephone technology applications can assist and facilitate a counselor to conduct counseling sessions more effectively. However, there are limitations and constraints in the implementation of this 'online' counseling which include aspects of confidentiality, non-verbal communication, professionalism, and cross-cultural practice. According to the statement from the respondents, there were concerns about the use of telephone in counseling in terms of professionalism where confidentiality issues were a major concern among respondents.

R1 expressed concern regarding unethical counselors who recorded the conversations during counseling session without seeking the client's permission and consent then disseminates the conversation. In addition, the use of location during the counseling process also plays crucial role in order to maintain the confidentiality of the issue discussed. While using tele-counseling services, respondents suggested that therapeutic places should be used during the counseling session, which requires special rooms such as face-to-face counseling so that the sessions will be able to run without any interruption and to maintain the confidentiality of the conversation.

.... examples are the same in terms of confidentiality if faced with an unethical counselor.

In terms of tele-counseling, counselor needs to explain in detail, seeking for the client's

permission to record the conversation and if the client refuses to be recorded then as a

professional counselor, it is considered as unethical. For these kinds of cases, who knows

that maybe the recording can fall into someone else's hand or maybe can go viral. So, it is

very important for the counselor to be professional. Second is in terms of place or

location. Just like today, when we talk on the phone, everyone can hear us, right? Even

video calls can be viewed by others, which is one of the issues of client's confidentiality

and security. Even if through phone, they still require therapeutic places. Like face-to-face

counseling sessions, we have one place or one room to go for sessions where only

counselor and client will be there without any interruption from others...(R1)

Invasion from unscrupulous parties was also a concern of respondents to tele-counseling services to address issues of a client's breach in confidentiality and security.

...The issue of confidentiality may also be a concern due to the possibility of breach in

confidentiality in the event of irresponsible parties interrupting conversations between

the counselor and the client, other than the type of session being held everywhere,

especially when it is likely to be the client's call time. Then where should he go to take

the call? But if the counselor is professional, he will refuse to accept the call and will ask

to be called afterwards when he is done. Then, the session will be conducted at the

appropriate place, as well as the client's side, where he calls the counselor...

The element of confidentiality is vital in a counseling session to ensure that client's information is kept safe and secure. Confidentiality is fundamental to building trust in relationship between counselor and client (Corey, 2013). With the sophistication of today's technology, others can intrude and access all the activities that have been done (Tyler \& Sabella, 2004). The possibility that such information is misused by a third party will endanger the wellbeing or security of the client.

In addition, the counselor's willingness to play an important role in providing client supports services. The counselor should have a professional attitude that should be available in all aspects of providing client support services especially providing telephone services so as not to affect the client's welfare. As stated by R1 in the statement below;

...The same goes to the counselor's readiness. I mean, if you were busy doing your work then suddenly you receive a call to listen to other people's problem. Will you be able to

listen to them and help at that moment? As for me, I don't think so because I'm already busy with my work and if my clients need my help, I won't be able to completely focus on them $100 \%$, right? There may also be counselors who try to serve their clients while doing their work. For me, it is very unethical but that might happen especially to the 'walk-in' type of clients. That's why as I said before, counseling needs to be done face-

to-face so as not to affect the client's well-being....

The statement is supported by $\mathrm{R} 1$ stating that professionalism must be present in counselors in assisting clients. The readiness of the counselor to handle tele-counseling services is crucial to determine the effectiveness of the session. 
...so, that's tele-counseling, not that I want to say I like it but I have to treat it because when you are needed, you need to listen. That's it. But I don't have much experience with tele-counseling. But I was tired of treating the clients. They know my home phone number. I was busy that time. So tired of having to treat one client from Johor, but when they called me I have to take care of it. But I still think that the weaknesses of telecounseling is that we have to listen to the clients although at that time, the time and location are not suitable. Because I was tired at that time, so I think it's not the right time to listen to anything. I wish a lot of counselors would have the intention to help because counseling is a helping profession....

Non-verbal communication is also one of the potential issues of tele-counseling services. It is a deficiency that can affect the well-being of clients in need of counseling services because through the R 1 tele-counseling service, there is a possibility of misunderstanding due to the absence of non-verbal communication and just listening over the phone.
.. there are still many downsides and limitations in conducting the sessions with only
listening and not face-to-face. It's a bit complicated to me. As I said, there are many possibilities and one of them is the misunderstanding of making conclusions by simply listening without a face-to-face conversation to see any non-verbal communication that occurs. If I it were up to me, I didn't really know how to make a conclusion just by listening...

Misinterpretation is also a limitation that has been a concern among respondents in handling tele-counseling services as a result of language and age differences between counselors and clients.

... There may be interventions as when at the time he says it, the intention was different. So,

it could be misinterpreted. So, there is an issue where the counselors and clients have conversation through chat and text but it was misinterpreted by the clients, probably because of the different level. Maybe there are specific cases where for example, let say if the client is underage between age of 16,17, under 18 or 13, or 14, it may seem easy but it is risky for the counselor. We have seen many cases like this where we have to settle some cases. This happened when if the parents see the text, they might misinterpret the text and there were cases that led into police report and such. So, we have to deal with it and resolve the matter...

These limitations will interfere with the effectiveness of the session and jeopardize the client's well-being. It may even result in losing the client's trust in the counselor for counseling services.

...If a certain client has a complex issue that requires a lot of skill to resolve and may take some time, I would advise that it is not a good idea to solve it by phone. This is because, it requires a very critical process and we do not want to affect the client. The client has given his trust to help him and he believes that we can help him but if we do not fully understand him, it will have an effect on the session. Well, maybe we can misinterpret it because we only listen and we cannot monitor the non-verbal communication gestures of the clients. It can be misinterpreted and worsen the situation. We can also lose the client's trust in us and it may be difficult for the client to seek help through counseling services in the future.

The effectiveness of online counseling will occur only if the counselor has conducted online counseling session with client. The absence of emotional guidance which is the absence of the physical presence of the counselor and client will result in the absence of emotional guidance such as tone of voice, facial expression and body language. This leads to weakness in expressing the right emotions. Even misinterpretations may also occur. This is because a counselor cannot identify and assess the client's body language in online counseling (Kim \& Dear, 2013). Murphy, Dingwall, Greatbatch, Parker, and Watson (1998) stated that the absence of these non-verbal communication elements would render counselors scarce resources in interpreting clients. In fact, the possibility of counselors misinterpreting or misunderstanding the meaning of an issue that the client is trying to address may result in losing the client's trust in the counselor to assist him.

The statements on the ethical issues raised by the average respondent are in line with the guidelines issued by the American Psychological Association (1997) and Bloom (1997) who suggested that all counselors who are interested in conducting online services should focus on 13 issues as a guide to this, such as taking into consideration of all legal and ethical code, informing clients of encryption steps, informing clients of data retention, taking steps on web invaders (impostors), obtaining guardianship for underage clients (minors), taking appropriate steps in restricting the dissemination of client's information, imposing online counseling boundaries on clients, providing network to licensed web sites that practice counseling, providing recognition as professional counselor to clients, providing alternative web or online addresses for clients to contact in the event of off-line counseling, stating the type of issues are not suitable to be addressed online, informing the clients about the limitations of technical interruptions and providing protocols that can be used to overcome misunderstanding.

\section{Conclusion}

In short, it can be perceived from this study that the views of counseling experts in tele-counseling services in Malaysia show that there are advantages and limitations in the implementation of this service. It is practical and can be used as an alternative to counseling services. However, in addition to the advantages it offers counselors who intend to offer these services should also consider the potential limitations of its implementation. It is the counselor's responsibility to ensure that clients are assisted optimally without any violation of the ethics of professionalism outlined.

The internet technology era has opened the boundaries of online communication, and it has also provided an opportunity for improvement in the provision of counseling services to the community. By taking into account the 
limitations and aspects that need to be emphasized; today's counseling profession practitioners should take the opportunity to adapt to the potential of providing online services as an alternative to conventional methods of faceto-face service implementation.

\section{References}

American Counseling Association. (1999). Code of ethics and standard of practice. Washington: DC Author.

American Psychological Association. (1997). APA statement on services by telephone, teleconferencing, and internet. Retrieved from: http://www.apa.org/ethics/stmnto1.html. [Accessed 12 Julai 2019].

Bloom, J. W. (1997). NBCC webcounseling standards. Retrieved from http://www.members.tripod.com/tcp_2/TCP_2/nbcc_stn.html. [Accessed 12 July 2019].

Boy, A. V., \& Pine, G. J. (1968). The counselor in the schools: A conceptualization. Boston: Houghton Mifflin.

Civin, M. (2000). Male, female, e-mail, the struggle for relatedness in a paranoid society. New York: Other Press.

Corey, G. (2013). Theory and practice of counseling and psychotherapy. Canada: United State.

Creswell, J. W. (2014). Research design: Qualitative, quantitative, and mixed methods approaches (4th ed.). USA: Sage.

Dini, F. (2016). Thesis of counseling strategies based on multicultural community welfare in Malaysia. Selangor: Universiti Kebangsaan Malaysia.

Glaser, \& Strauss. (1967). The discovery of grounded theory: Strategies for qualitative research. Chicago: Adline.

Glaser., B. G., \& Peshkin. (1992). Basics of grounded theory. Chiago: Adline.

Hanley, T. (2009). The working alliance in online therapy with young people: Preliminary findings. British Journal of Guidance \& Counselling, 37(3), 257-269. Available at: https://doi.org/10.1080/03069880902956991.

Ithnin, N., \& Ibrahim, O. (2000). E-learning live (live e-learning) in virtual learning. Paper presented at the Education Convention, UTM 2000.

Kim, J., \& Dear, D. R. (2013). Workspace satisfaction: The privacy-communication trade-off in open-plan offices. Journal of Environmental Psychology, 36, 18-26. Available at: https://doi.org/10.1016/j.jenvp.2013.06.007.

Leibert, T., Archer, J. J., Munson, J., \& York, G. (2006). An exploratory study of client perceptions of internet counseling and the therapeutic alliance. Journal of Mental Health Counseling, 28(1), 69-83. Available at: https://doi.org/10.17744/mehc.28.1.foh37djrw89nv6vb.

Mejah, H., Bakar, A. Y. A., \& Amat, S. (2019). Online counseling as an alternative of new millennial helping services. International Journal of Academic Research in Business \& Social Sciences, 9(9), 40-54. Available at: https://doi.org/10.6007/ijarbss/v9-i9/6267.

Mohd Nor, M. (2000). Information age from the perspective of Islam. Thinkers, 1(1), 15-26.

Murphy, E., Dingwall, R., Greatbatch, D., Parker, S., \& Watson, P. (1998). Qualitative research methods in health technology assessment: A review of the literature. Southampton: Health Technology Assessment.

Nor Ba'yah, A. K., \& Yahaya, M. (2001). Wellness psychology: E-counseling as an immediate method of assistance in solving psychological problems. Journal of Psychology and Human Development, 17(1), 225-242.

Pelling, N. (2009). The use of email and internet in counselling and psychological services. What practitioners need to know. Couselling, Psychotheraphy and Health, 5(1), 1-15.

Poh, L., Jaladin, R., \& Abdullah, H. S. (2013). Understanding the two sides of online counseling and their ethical and legal ramifications. Procedia-Social and Behavioral Sciences, 103, 1243-1251. Available at: https://doi.org/10.1016/j.sbspro.2013.10.453.

Ramsden, D. (2011). Experience Telephone counsellors: Experiences of the physical environment in a non-face-to-face counselling servicees. Counselling Autralia, 12(3), 26-36.

Richards, D., \& Vigan'o, N. (2012). Online counseling. In Y. Zheng (Ed.), Encyclopedia of Cyber Behavior (Vol. 1, pp. 699-713). New York: IGI Global.

Rosenfield, M. (2002). Electronic technology for social work education and practice: The application of telephone technology to counseling. Journal of Technology in Human Services, 20(1-2), 173-181. Available at: https://doi.org/10.1300/jo17v20no1_13.

Suriati, G., \& Colonius, A. (2008). Query and interpretation of qualitative information in geography research: Experience from women's family welfare wellness review. Journal of Human and Society, 16(11), 156-179.

Tyler, M. J., \& Sabella, R. A. (2004). Using technology to improve counseling practice: A primer for the 21st century. Alexandria, VA: American Counseling Association.

Yin, R. K. (2014). Case study research: Design and methods (5th ed.). Thousand Oaks, California: SAGE Publications Inc.

Young, K. S. (2005). An empirical examination of client attitudes towards online counselling. Cyber Psychology \& Behavior, 8(2), $172-177$. Available at: https://doi.org/10.1089/cpb.2005.8.172. 